\title{
Functional Geominimal Surface Area and Its Related Affine Isoperimetric Inequality
}

\author{
Niufa Fang $\mathbb{D}^{1}$ and Jin Yang ${ }^{2}$ \\ ${ }^{1}$ Chern Institute of Mathematics, Nankai University, Tianjin 300071, China \\ ${ }^{2}$ Department of Mathematics, Hubei Minzu University, Enshi, Hubei 445000, China \\ Correspondence should be addressed to Niufa Fang; fangniufa@nankai.edu.cn
}

Received 13 June 2020; Accepted 29 June 2020; Published 13 July 2020

Academic Editor: Chang-Jian Zhao

Copyright (c) 2020 Niufa Fang and Jin Yang. This is an open access article distributed under the Creative Commons Attribution License, which permits unrestricted use, distribution, and reproduction in any medium, provided the original work is properly cited.

\begin{abstract}
The first variation of the total mass of log-concave functions was studied by Colesanti and Fragalà, which includes the $L_{p}$ mixed volume of convex bodies. Using Colesanti and Fragalà's first variation formula, we define the geominimal surface area for logconcave functions, and its related affine isoperimetric inequality is also established.
\end{abstract}

\section{Introduction}

As we have known, Minkowski addition (the vector addition of convex bodies) is the cornerstone in the classical BrunnMinkowski theory. Combining with volume, it leads to the Brunn-Minkowski inequality that is one of the most important results in convex geometry. The first variation of volume with respect to Minkowski addition is named the first mixed volume, and its related inequality is the Minkowski inequality. For more history and developments of the BrunnMinkowski inequality, one may refer to the excellent survey [1]. For instance, the Prékopa-Leindler inequality [2-8] is known as the functional version of the Brunn-Minkowski inequality. In recent years, finding the functional counterparts of existing geometric results, especially for logconcave functions, has been receiving intensive attentions (see, e.g., [9-34]).

In 2013, Colesanti and Fragalà [35] introduced the "Minkowski addition" and "scalar multiplication," $\alpha \cdot f \oplus \beta \cdot g$ (where $\alpha, \beta>0$ ), of log-concave functions $f$ and $g$ as

$$
\alpha \cdot f \oplus \beta \cdot g(x)=\sup _{y \in \mathbb{R}^{n}} f\left(\frac{x-y}{\alpha}\right)^{\alpha} g\left(\frac{y}{\beta}\right)^{\beta} .
$$

We remark that a function $f: \mathbb{R}^{n} \longrightarrow[0, \infty)$ is $\log$ concave if it has the form $f(x)=e^{-u(x)}$, where $u: \mathbb{R}^{n} \longrightarrow \mathbb{R}$ $\cup\{+\infty\}$ is convex. The total mass of $f$ is defined as

$$
J(f)=\int_{\mathbb{R}^{n}} f(x) d x
$$

Similar to the case of convex bodies, Colesanti and Fragalà [35] considered the following variational

$$
\delta J(f, g)=\lim _{t \rightarrow 0^{+}} \frac{J(f \oplus t \cdot g)-J(f)}{t}
$$

and it is called the first variation of $J$ at $f$ along $g$. The first variation, $\delta J(f, g)$, includes the $L_{p}$ mixed volume when it restricted $f$ and $g$ to the subclass of log-concave functions (see [35], Proposition 3.12).

Colesanti and Fragalà's work inspired us a natural way to extend the $L_{p}$ geominimal surface area for convex bodies to the class of log-concave functions. For convenience, we recall the definition of $L_{p}$ geominimal surface area. For a convex body $K$ containing the origin in its interior, its $L_{p}$ geominimal surface area, $G_{p}(K)$, is defined as (the case $p=1$, see Petty [36], and $p>1$, see Lutwak [37])

$$
\omega_{n}^{p / n} G_{p}(K)=\inf \left\{n V_{p}(K, Q) V\left(Q^{\circ}\right)^{p / n}: Q \in \mathscr{K}_{o}^{n}\right\}
$$


where $\omega_{n}$ is the volume of the unit ball in $n$-dimensional Euclidean space $\mathbb{R}^{n}, Q^{\circ}$ is the polar body of $Q$ defined by $Q^{\circ}=\left\{x \in \mathbb{R}^{n}:\langle x, y\rangle \leq 1, \forall y \in Q\right\}, \mathscr{K}_{0}^{n}$ denotes the class of convex bodies in $\mathbb{R}^{n}$ that contain the origin in their interiors, and $V_{p}(K, Q)$ is the $L_{p}$ mixed volume (for detailed definition, see Section 2). The fundamental inequality for $L_{p}$ geominimal surface area is the following affine isoperimetric inequality (see, e.g., [37], Theorem 3.12):

$$
G_{p}(K)^{n} \leq n^{n} \omega_{n}^{p} V(K)^{n-p},
$$

with equality if and only if $K$ is an ellipsoid.

The $L_{p}$ geominimal surface area, $G_{p}(K)$, is an important notation in the $L_{p}$ Brunn-Minkowski theory, which serves as a bridge connecting affine differential geometry, relative differential geometry, and Minkowski geometry. In the past three decades, the $L_{p}$ geominimal surface area has developed rapidly (see $[25,38-42]$ for some of the pertinent results).

Since $\delta J(f, g)$ includes the $L_{p}$ mixed volume, we extend the $L_{p}$ geominimal surface area to the functional version as follows.

Definition 1. Let $f: \mathbb{R}^{n} \longrightarrow[0, \infty)$ be an integral log-concave function and $p>0$. The $L_{p}$ geominimal surface area of $f$ is defined as

$c_{n}^{p / n} G_{p}(f)=\inf \left\{\delta J(f, g) J\left(g^{\circ}\right)^{p / n}: g\right.$ is a log-concave function $\}$,

where $c_{n}=(2 \pi)^{n / 2}$, and $g^{\circ}(x)=\inf _{y \in \mathbb{R}^{n}} e^{-\langle x, y\rangle} / g(y)$ is the polar function of $g$.

In Lemma 5, we prove that the above definition includes the $L_{p}$ geominimal surface area (4) when $p \geq 1$ and restricted $f, g$ to the subclass of log-concave functions.

In order to study the functional geominimal surface area, we need the integral formula of $\delta J(\cdot, \cdot)$. Hence, we need some notations. We write $\langle x, y\rangle$ for the usual inner product of $x, y \in \mathbb{R}^{n}$, and $\|x\|$ denotes the Euclidean normal of $x \in$ $\mathbb{R}^{n}$. We say that $g=e^{-v}$ is an admissible perturbation for $f=e^{-u}$ if there exists a constant $c>0$ such that $u^{*}-c v^{*}$ is convex, where $u^{*}(y)=\sup _{x \in \mathbb{R}^{n}}\{\langle x, y\rangle-u(x)\}$ is the Legendre conjugate of $u$. Let $\mathscr{A}^{\prime}$ denote the set of logconcave functions given by function $f$ such that $u=-\log$ $f$ belongs to

$\mathscr{L}^{\prime}=\left\{u \in \mathscr{L}: \operatorname{dom}(u)=\mathbb{R}^{n}, u \in \mathscr{C}_{+}^{2}\left(\mathbb{R}^{n}\right), \lim _{\|x\| \rightarrow+\infty} \frac{u(x)}{\|x\|}=+\infty\right\}$.

Here, $\operatorname{dom}(u)=\left\{x \in \mathbb{R}^{n}: u(x)<+\infty\right\}$ and

$\mathscr{L}=\left\{u: \mathbb{R}^{n} \longrightarrow \mathbb{R} \cup\{+\infty\} \mid \quad u\right.$ is convex, $\left.\operatorname{dom}(u) \neq \varnothing, \quad \lim _{\|x\| \rightarrow+\infty} u(x)=+\infty\right\}$.
Colesanti and Fragalà ([35], Theorem 4.5) provided an integral formula for the first variation $\delta J(f, g)$ when $f, g$ $\in \mathscr{A}^{\prime}$ and $g$ is an admissible perturbation for $f$. For our aims, we consider the following optimization problem:

$\inf \left\{\delta J(f, g) J\left(g^{\circ}\right)^{p / n}: f, g \in \mathscr{A}^{\prime}, p>0\right.$ and $g$ is an admissible perturbation for $\left.f\right\}$.

If the extremum in (9) exists, then it is denoted by $c_{n}^{p / n} G_{p}^{(1)}(f)$.

In Section 3, we prove that for $p>0$ and $f \in \mathscr{A}^{\prime}$, if $J(f)$ is finite, then there exists a unique $\log$-concave function $\bar{f} \in \mathscr{A}^{\prime}$ such that

$$
G_{p}^{(1)}(f)=\delta J(f, \bar{f}) \text { and } J\left(\bar{f}^{\circ}\right)=c_{n} .
$$

Similar to the geometric case, the unique log-concave function $\bar{f}$ is called $p$-Petty functions of $f$ and denoted by $T_{p} f$.

Using $p$-Petty functions, we obtain the following analytic inequality with equality conditions involving $G_{p}^{(1)}(f)$.

Theorem 2. Suppose $f \in \mathscr{A}^{\prime}$ and $p>0$. If $f$ has its barycenter at 0 (i.e., $\int_{\mathbb{R}^{n}} x f(x) d x=0$ ), then

$$
J(f)^{p / n} G_{p}^{(1)}(f) \leq c_{n}^{p / n}\left(n J(f)+\int_{\mathbb{R}^{n}} f \log f d x\right),
$$

with equality if $T_{p} f(x)=f(x)$ and $f(x)=c e^{-\left(\|A x\|^{2} / 2\right)}$ for $A \epsilon$ $S L(n)$ and $c>0$.

\section{Background}

2.1. Functional Setting. Let $u: \mathbb{R}^{n} \longrightarrow \mathbb{R} \cup\{+\infty\}$ if for every $x, y \in \mathbb{R}^{n}$ and $\lambda \in[0,1]$ it satisfies

$$
u((1-\lambda) x+\lambda y) \leq(1-\lambda) u(x)+\lambda u(y),
$$

we say $u$ is a convex function; let

$$
\operatorname{dom}(u)=\left\{x \in \mathbb{R}^{n}: u(x) \in \mathbb{R}\right\} .
$$

By the convexity of $u$, dom $(u)$ is a convex set. We say that $u$ is proper if $\operatorname{dom}(u) \neq \varnothing$. The Legendre conjugate of $u$ is the convex function defined by

$$
u^{*}(y)=\sup _{x \in \mathbb{R}^{n}}\{\langle x, y\rangle-u(x)\} \quad \forall y \in \mathbb{R}^{n} .
$$

Clearly, $u(x)+u^{*}(y) \geq\langle x, y\rangle$ for all $x, y \in \mathbb{R}^{n}$; there is an equality if and only if $x \in \operatorname{dom}(u)$ and $y$ is in the subdifferential of $u$ at $x$. Hence, it can be checked that

$$
u^{*}(\nabla u(x))+u(x)=\langle x, \nabla u(x)\rangle .
$$

On the class of convex functions from $\mathbb{R}^{n}$ to $\mathbb{R} \cup\{+\infty\}$, 
the infimal convolution is defined by

$$
u \square v(x)=\inf _{y \in \mathbb{R}^{n}}\{u(x-y)+v(y)\} \quad \forall x \in \mathbb{R}^{n},
$$

and the right scalar multiplication by a nonnegative real number $\alpha>0$,

$$
(u \alpha)(x)=\alpha u\left(\frac{x}{\alpha}\right)
$$

It was proved in [21] (Proposition 2.1) that if $u, v: \mathbb{R}^{n}$ $\longrightarrow \mathbb{R} \cup\{+\infty\}$ are convex functions and $\alpha>0$, then

$$
\begin{gathered}
(u \square v)^{*}=u^{*}+v^{*}, \\
(u \alpha)^{*}=\alpha u^{*} .
\end{gathered}
$$

The following result will be used later.

Theorem 3 ([43], Theorem 10.9). Let $C$ be a relatively open convex set, and let $f_{1}, f_{2}, \cdots$ be a sequence of finite convex functions on C. Suppose that the real number $f_{1}(x), f_{2}(x), \cdots$ is bounded for each $x \in C$. It is then possible to select a subsequence of $f_{1}, f_{2}, \cdots$, which converges uniformly on closed bounded subsets of $C$ to some finite convex function $f$.

The functional Blaschke-Santaló inequality states that let $f, g$ be nonnegative integrable functions on $\mathbb{R}^{n}$ satisfying

$$
f(x) g(y) \leq e^{-\langle x, y\rangle}, \quad \forall x, y \in \mathbb{R}^{n} .
$$

If $f$ has its barycenter at 0 , which means that $\int_{\mathbb{R}^{n}} x f(x)$ $d x=0$, then

$$
\left(\int_{\mathbb{R}^{n}} f(x) d x\right)\left(\int_{\mathbb{R}^{n}} g(x) d x\right) \leq c_{n}^{2}
$$

with equality if and only if there exists a positive definite matrix $A$ and $C>0$ such that, a.e. in $\mathbb{R}^{n}$,

$$
f(x)=C e^{-\frac{\langle A x, x\rangle}{2}}, \quad g(y)=C^{-1} e^{-\frac{\left\langle A^{-1} x, x\right\rangle}{2}} .
$$

2.2. The First Variation of the Total Mass of Log-Concave Functions. In this paper, we set

$$
\begin{aligned}
& \mathscr{L}=\left\{u: \mathbb{R}^{n} \longrightarrow \mathbb{R} \cup\{+\infty\} \mid \text { u proper, convex, } \lim _{\|x\| \rightarrow+\infty} u(x)=+\infty\right\}, \\
& \mathscr{A}=\left\{f: \mathbb{R}^{n} \longrightarrow \mathbb{R} \mid f=e^{-u}, u \in \mathscr{L}\right\} .
\end{aligned}
$$

The total mass functional of $f$ is defined as

$$
J(f)=\int_{\mathbb{R}^{n}} f(x) d x
$$

The Gaussian function

$$
\gamma(x)=e^{-\frac{\|x\|^{2}}{2}}
$$

plays within class $\mathscr{A}$ the role of the ball in the set of convex bodies, and $J(\gamma)=(2 \pi)^{n / 2}=c_{n}$. For every $A \in \mathrm{GL}(n)$, we write

$$
\gamma_{A}(x)=e^{-\frac{\|A x\|^{2}}{2}}
$$

From the definition of polar function and Legendre conjugate of function, we note that if $f \in \mathscr{A}$, then

$$
f^{\circ}=e^{-\varphi^{*}}
$$

The support function of log-concave function $f=e^{-\varphi}$ is (see [44])

$$
h_{f}(x)=\varphi^{*}(x) \text {. }
$$

This is a proper generalization, in the sense that $h_{\chi_{K}}=h_{K}$. Let $f=e^{-u}, g=e^{-v}$, and let $\alpha, \beta>0$, then

$$
\alpha \cdot f \oplus \beta \cdot g=e^{-[(u \alpha) \square(v \beta)],}
$$

which in explicit form reads

$$
(\alpha \cdot f \oplus \beta \cdot g)(x)=\sup _{y \in \mathbb{R}^{n}} f\left(\frac{x-y}{\alpha}\right)^{\alpha} g\left(\frac{y}{\beta}\right)^{\beta} .
$$

The support function of $\alpha \cdot f \oplus \beta \cdot g$ satisfies

$$
h_{\alpha \cdot f \oplus \beta \cdot g}(x)=\alpha h_{f}(x)+\beta h_{g}(x) \text {. }
$$

In particular,

$$
h_{\alpha \cdot f}(x)=\alpha h_{f}(x) \text {. }
$$

Let $f, g \in \mathscr{A}$. The first variation of $J$ at $f$ along $g$ is defined as

$$
\delta J(f, g)=\lim _{t \rightarrow 0^{+}} \frac{J(f \oplus t \cdot g)-J(f)}{t} .
$$

The existence of the above limit was proved by Colesanti and Fragalà [35], and $\delta J(f, g) \in[-k,+\infty]$ with $k=\max \{$ inf $(-\log g), 0\} J(f)$. In particular, for every $f \in \mathscr{A}$ with $J(f)>$ 0 , then

$$
\delta J(f, f)=n J(f)+\int_{\mathbb{R}^{n}} f \log f d x .
$$

The functional version of Minkowski first inequality reads as follows (see, e.g., [35], Theorem 5.1): let $f, g \in \mathscr{A}$ and assume that $J(f)>0$. Then,

$$
\delta J(f, g) \geq J(f)\left[\log \frac{J(g)}{J(f)}+n\right]+\int_{\mathbb{R}^{n}} f \log f d x,
$$

with equality if and only if there exists $x_{0} \in \mathbb{R}^{n}$ such that $g(x)=f\left(x-x_{0}\right)$ for $\forall x \in \mathbb{R}^{n}$. 
Let $\mathscr{K}^{n}$ denote the set of convex bodies (compact, convex subsets with nonempty interiors) in the Euclidean space $\mathbb{R}^{n}$. We write $\mathscr{K}_{o}^{n}$ for the set of convex bodies that contain the origin in their interiors. Let $V(K)$ denote the $n$-dimensional volume of convex body $K$. The volume of the standard unit ball in $\mathbb{R}^{n}$ is denoted by $\omega_{n}=\pi^{n / 2} / \Gamma((n / 2)+1)$. A convex body $K \in \mathscr{K}^{n}$ is uniquely determined by its support function, which is defined as $h_{K}(x)=\max \{\langle x, y\rangle: y \in K\}$, where $\langle\cdot, \cdot\rangle$ denotes the usual inner product in $\mathbb{R}^{n}$. The polar body of $K$ is defined by $K^{\circ}=\left\{x \in \mathbb{R}^{n}:\langle x, y\rangle \leq 1, \forall y \in K\right\}$.

For real $p \geq 1, K, L \in \mathscr{K}^{n}$, and real $\varepsilon>0$, the MinkowskiFirey $L_{p}$ combination $K{ }_{p} \varepsilon \cdot L$ is a convex body whose support function is given by

$$
h\left(K+_{p} \varepsilon \cdot L, \cdot\right)^{p}=h(K, \cdot)^{p}+\varepsilon h(L, \cdot)^{p} .
$$

The $L_{p}$ mixed volume $V_{p}(K, L)$ of convex bodies $K$ and $L$ is defined by

$$
V_{p}(K, L)=\frac{p}{n} \lim _{\varepsilon \rightarrow 0^{+}} \frac{V\left(K+_{p} \varepsilon \cdot L\right)-V(K)}{\varepsilon} .
$$

The existence of this limit is showed in [45].

The following result show that $\delta J(f, g)$ includes the $L_{p}$ mixed volume for convex bodies.

Proposition 4 ([35], Proposition 3.12). Let $q \in(1,+\infty), p=$ $q /(q-1)$ and $K, L \in \mathscr{K}_{o}^{n}$. Let $u=\left(h_{K^{\circ}}(x)^{q}\right) / q, v(x)=\left(h_{L^{\circ}}\right.$ $\left.(x)^{q}\right) / q$, and $f=e^{-u}, g=e^{-v}$. Then, there exists a positive constant $c=c(n, q)$ such that

$$
J(f)=c(n, q) V(K)
$$

with $c(n, q)=q^{n / q} \Gamma((n+q) / q)$, and

$$
\frac{p}{n} \delta J(f, g)=c(n, q) V_{p}(K, L) .
$$

We set $\mathscr{A}^{\prime}$ as the subclasses of $\mathscr{A}$ given by the function $f$ such that $u=\log f$ belongs to

$$
\mathscr{L}^{\prime}=\left\{u \in \mathscr{L}: \operatorname{dom}(u)=\mathbb{R}^{n}, \quad u \in \mathscr{C}_{+}^{2}\left(\mathbb{R}^{n}\right), \quad \lim _{\|x\| \rightarrow+\infty} \frac{u(x)}{\|x\|}=+\infty\right\} .
$$

For log-concave function $f=e^{-u}$, the Borel measure $\mu_{f}$ on $\mathbb{R}^{n}$ is defined by (see [35])

$$
\mu_{f}=(\nabla u)_{\#}\left(f \mathscr{H}^{n}\right) .
$$

Here, $\mathscr{H}^{n}$ is the $n$-dimensional Hausdorff measure. We need the fact that the barycenter of $\mu_{f}$ is the origin; i.e.,

$$
\int_{\mathbb{R}^{n}} x \mu_{f}(x)=0
$$

We recall that the log-concave function $g=e^{-v}$ is an admissible perturbation for log-concave function $f=e^{-u}$ if

$$
\exists a>0: u^{*}-a v^{*}
$$

is convex.

Colesanti and Fragalà [35] provided an integral representation of the first variation $\delta J(\cdot, \cdot)$ (see, e.g., [35], Theorem 4.5): let $f=e^{-u}$ and $g=e^{-v} \in \mathscr{A}^{\prime}$ and assume that $g$ is an admissible perturbation for $f$. Then, $\delta J(f, g)$ is finite and is given by

$$
\delta J(f, g)=\int_{\operatorname{dom}(u)} h_{g}(x) d \mu_{f}(x) .
$$

\section{Functional $L_{p}$ Geominimal Surface Areas}

Analogy to convex bodies, for $f \in \mathscr{A}$ and $p \in \mathbb{R}$, we consider the following optimization problem:

$$
c_{n}^{p / n} G_{\lambda}(f)=\inf \left\{\delta J(f, g) J\left(g^{\circ}\right)^{p / n}: g \in \mathscr{A}\right\}
$$

The following result shows that the above optimization problem includes Lutwak's $L_{p}$ geominimal surface areas for convex bodies (4) when $p>1$ (up to a constant which is dependent onnand $p$ ). This is one of the reasons why $G_{p}(f)$ is called the $L_{p}$ geominimal surface area for log-concave function $f$.

Lemma 5. Let $p>1, q=p /(p-1)$, and $K \in \mathscr{K}_{o}^{n}$. Iff $=e^{-h_{K^{\circ}(x)}^{q} / q}$, then

$$
G_{p}(f)=\alpha(n, p) G_{p}(K)
$$

with $\alpha(n, p)=(1 / p) c(n, q) c(n, p)^{p / n} \omega_{n}^{p / n} c_{n}^{-(p / n)}$ for $c(n, q)=$ $q^{n / q} \Gamma((n+q) / q)$.

Proof. Let $K, L \in \mathscr{K}_{o}^{n}, u(x)=h_{K^{\circ}}(x)^{q} / q, v(x)=h_{L^{\circ}}(x)^{q} / q$, and $f=e^{-u}, g=e^{-v}$. It is not hard to see that

$$
v^{*}(x)=\frac{h_{L}(x)^{p}}{p}
$$

Then, Proposition 4 tells us that

$$
\begin{gathered}
J\left(g^{\circ}\right)=c(n, p) V\left(L^{\circ}\right), \\
\frac{p}{n} \delta J(f, g)=c(n, q) V_{p}(K, L),
\end{gathered}
$$

with $c(n, q)=q^{n / q} \Gamma((n+q) / q)$. 
From the definitions of $L_{p}$ geominimal surface area of convex bodies (4) and log-concave functions (44), we have

$$
\begin{aligned}
c_{n}^{p / n} & G_{p}(f)=\inf \left\{\delta J(f, g) J\left(g^{o}\right)^{p / n}: g \in \mathscr{A}\right\} \\
\quad= & \inf \left\{\frac{n}{p} c(n, q) c(n, p)^{p / n} V_{p}(K, L) V\left(L^{\circ}\right)^{p / n}: K, L \in \mathscr{K}_{o}^{n}\right\} \\
\quad= & \frac{1}{p} c(n, q) c(n, p)^{p / n} \omega_{n}^{p / n} G_{p}(K) .
\end{aligned}
$$

Since we need the integral representation of the first variation $\delta J(f, g)$ in dealing the problem (44), we focus on

$c_{n}^{p / n} G_{p}^{(1)}(f)=\inf \left\{\delta J(f, g) J\left(g^{\circ}\right)^{p / n}: g \in \mathscr{A}^{\prime}\right.$ and $g$ is an admissible perturbation for $\left.\mathrm{f}\right\}$

for $f \in \mathscr{A}^{\prime}$ and $p \in \mathbb{R}$. Trivially, $G_{p}(f) \leq G_{p}^{(1)}(f)$.

We need the next lemma.

Lemma 6. Let $f, g \in \mathscr{A}^{\prime}$ and assume that $g$ is an admissible perturbation for $f$. If $A \in S L(n)$, then

$$
\delta J(f \circ A, g \circ A)=\delta J(f, g) .
$$

Proof. Let $f(x)=e^{-u(x)}$ and $g(x)=e^{-v(x)}$. We note that

$$
\begin{aligned}
(v \circ A)^{*}(x) & =\sup _{y \in \mathbb{R}^{n}}\{\langle x, y\rangle-v(A y)\} \\
& =\sup _{y \in \mathbb{R}^{n}}\left\{\left\langle A^{-t} x, A y\right\rangle-v(A y)\right\}=v^{*}\left(A^{-t} x\right) .
\end{aligned}
$$

Since $\nabla_{x}(u \circ A)=A^{t} \nabla_{A x} u$, we have

$$
\begin{aligned}
\delta J(f \circ A, g \circ A) & =\int_{\mathbb{R}^{n}}(v \circ A)^{*}(\nabla(u \circ \mathrm{A})(x)) f \circ A(x) d x \\
& =\int_{\mathbb{R}^{n}}(v \circ A)^{*}\left(A^{t} \nabla_{A x} u(A x)\right) f(A x) d x \\
& =\int_{\mathbb{R}^{n}} v^{*}\left(\nabla_{A x} u(A x)\right) f(A x) d \\
& =\int_{\mathbb{R}^{n}} v^{*}(\nabla u(z)) f(z) d z=\delta J(f, g) .
\end{aligned}
$$

The following result shows that the functional geominimal surface area is affine invariant.

Lemma 7. Suppose $f \in \mathscr{A}^{\prime}$ and $p>0$. If $A \in \operatorname{SL}(n)$, then

$$
G_{p}^{(1)}(f \circ A)=G_{p}^{(1)}(f) .
$$

Proof. By (51) and the definition of polar function (26), we have

$$
J\left((g \circ A)^{\circ}\right)=J\left(g^{\circ} \circ A^{-t}\right)=J\left(g^{\circ}\right),
$$

for $A \in \mathrm{SL}(n)$. Combing with Lemma 6, we have

$$
\delta J(f \circ A, g) J\left(g^{\circ}\right)^{p / n}=\delta J\left(f, g \circ A^{-1}\right) J\left(\left(\left(g \circ A^{-1}\right)^{\circ}\right)\right)^{p / n} .
$$

Therefore, we obtain

$$
G_{p}^{(1)}(f \circ A)=G_{p}^{(1)}(f),
$$

for $A \in \operatorname{SL}(n)$.

The following lemma was proved by Cordero-Erausquin and Klartag ([46], Lemma 16).

Lemma 8. Let $\mu$ be a finite Borel measure in $\mathbb{R}^{n}$, and let $K$ be the interior of $\operatorname{conv}(\operatorname{Supp}(\mu))$. If $x_{0} \in K$ and the barycenter of $\mu$ lies at the origin, then there exists a constant $C_{\mu, x_{0}}>0$ with the following property: for any nonnegative, $\mu$-integrable, convex function $\varphi: \mathbb{R}^{n} \longrightarrow \mathbb{R} \cup\{+\infty\}$,

$$
\varphi\left(x_{0}\right) \leq C_{\mu, x_{0}} \int_{\mathbb{R}^{n}} \varphi d \mu .
$$

The next proposition shows that the infimum in the definition of the $p$-geominimal surface area of log-concave function is a minimum.

Proposition 9. Let $p>0$ and $f \in \mathscr{A}^{\prime}$. If $J(f)$ is finite, then there exists a unique log-concave function $\bar{f} \in \mathscr{A}$ such that

$$
G_{p}^{(1)}(f)=\delta J(f, \bar{f}) \text { and } J\left(\bar{f}^{\circ}\right)=c_{n} .
$$

Proof. From the definition of $G_{p}^{(1)}(f)$, there exists a sequence $g_{i} \in \mathscr{A}^{\prime}$ such that $J\left(g_{i}^{\circ}\right)=c_{n}$, with $\delta J(f, \gamma) \geq \delta J\left(f, g_{i}\right)$ for all $i$, and

$$
\delta J\left(\mathrm{f}, g_{i}\right) \longrightarrow G_{p}^{(1)}(f) .
$$

Let $g_{i}(x)=e^{-v_{i}(x)}$, then

$$
\delta J(f, \gamma) \geq \delta J\left(f, g_{i}\right)=\int_{\mathbb{R}^{n}} v_{i}^{*}(x) d \mu_{f}(x) .
$$

First, we assume that $v_{i}$ are nonnegative and $v_{i}(0)=0$ for all $i$. In this case, from (14), we have

$$
v_{i}^{*}(x)=\sup _{y \in \mathbb{R}^{n}}\left\{\langle y, x\rangle-v_{i}(y)\right\} \geq\langle 0, x\rangle-v_{i}(0)=0,
$$

and

$$
v_{i}^{*}(0)=\sup _{y \in \mathbb{R}^{n}}\left\{\langle y, 0\rangle-v_{i}(y)\right\}=-\inf _{y \in \mathbb{R}^{n}} v_{i}(y)=0 .
$$

Let $K$ be the interior ofconv $\left(\operatorname{Supp}\left(\mu_{f}\right)\right)$. By Lemma 8 and ((59)), we conclude that $v_{i}^{*}$ are uniformly upper bound which 
is dependent only onf. According to Theorem 3, there exists a subsequence $\left\{v_{i_{j}}^{*}\right\}_{j=1,2 \ldots}$ that converges pointwise in $K$ to a convex function $v^{*}: K \longrightarrow \mathbb{R}$. We extend the definition of $v^{*}$ by setting $v^{*}(x)=+\infty$ for $x \in \bar{K}$ and for $x \in \partial K$,

$$
v^{*}(x)=\lim _{\lambda \rightarrow 1^{-}} v^{*}(\lambda x)
$$

This limit always exists in $[0,+\infty]$, since the function $\lambda$ $\mapsto v^{*}(\lambda x)$ is nondecreasing for $\lambda \in(0,1)$ following from the convexity of $v^{*}$ and $v^{*}(0)=0$. Moreover, we have that $v^{*}(\lambda$ $x) \mathrm{Z} v^{*}(x)$ as $\lambda \longrightarrow 1^{-}$for any $x \in \bar{K}$. Because $v_{i}^{*} \longrightarrow v^{*}$ is equivalent to $v_{i} \longrightarrow v$ (here, $\left.v=\left(v^{*}\right)^{*}\right)$, hence, there exits a log-concave function $\bar{f}=e^{-v}$ which satisfies the claim.

In the general case, there exist $x_{0}^{(i)} \in \mathbb{R}^{n}$ and $\inf _{x \in \mathbb{R}^{n}} v_{i}(x)$ $=d_{i} \in \mathbb{R}$ such that $\bar{v}_{i}(x)=v_{i}\left(x-x_{0}^{(i)}\right)-d_{i}$ are nonnegative and $\bar{v}_{i}(0)=0$ for all $i=1,2, \cdots$. The convexity of $v_{i}$ and $e^{-v_{i}}$ $\in \mathscr{A}^{\prime}$ ensures the finiteness of $d_{i}$; i.e., $\left|d_{i}\right|<k$ for some $k>0$. Similar to the first case, we have

$$
\delta J(f, \gamma) \geq \delta J\left(f, \bar{g}_{i}\right)=\int_{\mathbb{R}^{n}} \bar{v}_{i}^{*}(x) d \mu_{f}(x),
$$

where $\bar{g}_{i}=e^{-v_{i}}$. Lemma 8 deduces that

$$
\delta J(f, \gamma) \geq \delta J\left(f, \bar{g}_{i}\right)=\int_{\mathbb{R}^{n}} \bar{v}_{i}^{*}(x) d \mu_{f}(x) \geq \frac{1}{C_{\mu_{f}, x_{0}}} \bar{v}_{i}^{*}\left(x_{0}\right)
$$

holds for $x_{0} \in K$. Moreover,

$$
\begin{aligned}
\bar{v}_{i}^{*}(x) & =\sup _{y \in \mathbb{R}^{n}}\left\{\langle y, x\rangle-\bar{v}_{i}(y)\right\} \\
= & \sup _{y \in \mathbb{R}^{n}}\left\{\langle y, x\rangle-v_{i}\left(y-x_{0}^{(i)}\right)\right\}+d_{i} \\
= & \sup _{y \in \mathbb{R}^{n}}\left\{\left\langle y+x_{0}^{(i)}, x\right\rangle-v_{i}(y)\right\}+d_{i} \\
= & v_{i}^{*}(x)+\left\langle x, x_{0}^{(i)}\right\rangle+d_{i} .
\end{aligned}
$$

Therefore,

$$
C_{\mu_{f}, x_{0}} \delta J(f, \gamma) \geq \bar{v}_{i}^{*}\left(x_{0}\right)=v_{i}^{*}\left(x_{0}\right)+\left\langle x_{0}, x_{0}^{(i)}\right\rangle+d_{i},
$$

i.e.,

$$
v_{i}^{*}\left(x_{0}\right) \leq C_{\mu_{f}, x_{0}} \delta J(f, \gamma)-\left\langle x_{0}, x_{0}^{(i)}\right\rangle-d_{i}
$$

for any $x_{0} \in K$. Then, along the same line of the first case, we conclude that the claim of this proposition holds.

The uniqueness of the minimizing function is demonstrated as follows. Suppose $h_{1}, h_{2} \in \mathscr{A}$, such that $J\left(h_{1}^{\circ}\right)=J($ $\left.h_{2}^{\circ}\right)=c_{n}$, and

$$
\begin{aligned}
\delta J\left(f, h_{1}\right) J\left(h_{1}^{\circ}\right)^{p / n} & =\inf \left\{\delta J(f, g) J\left(g^{\circ}\right)^{p / n}: g \in \mathscr{A}^{\prime}\right\} \\
& =\delta J\left(f, h_{2}\right) J\left(h_{2}^{\circ}\right)^{p / n},
\end{aligned}
$$

i.e.,

$$
\delta J\left(f, h_{1}\right)=\delta J\left(f, h_{2}\right) .
$$

Let $h_{1}=e^{-v_{1}}$ and $h_{2}=e^{-v_{2}}$. Define $h \in \mathscr{A}^{\prime}$, by

$$
h=\frac{1}{2} \cdot h_{1} \oplus \frac{1}{2} \cdot h_{2}=e^{-v_{1}(1 / 2) \square v_{2}(1 / 2)} .
$$

Then, from (18) and (70), we have

$$
\begin{aligned}
\delta J(f, h) & =\int_{\mathbb{R}^{n}}\left(v_{1} \frac{1}{2} \square v_{2} \frac{1}{2}\right)^{*}(x) d \mu_{f}(x) \\
& =\frac{1}{2} \int_{\mathbb{R}^{n}} v_{1}^{*}(x) d \mu_{f}(x)+\frac{1}{2} \int_{\mathbb{R}^{n}} v_{2}^{*}(x) d \mu_{f}(\mathrm{x}) \\
& =\frac{1}{2} \delta J\left(f, h_{1}\right)+\frac{1}{2} \delta J\left(f, h_{2}\right)=\delta J\left(f, h_{1}\right)=\delta J\left(f, h_{2}\right),
\end{aligned}
$$

and by the basic inequality $\sqrt{a b} \leq(a+b) / 2$ for $a, b>0$ and (18), we have

$$
\begin{aligned}
J\left(h^{\circ}\right)= & \int_{\mathbb{R}^{n}} e^{-\left[\left(v_{1} 1 / 2\right) \square\left(v_{2}(1 / 2)\right)\right]^{*}} d x=\int_{\mathbb{R}^{n}} e^{-\left[(1 / 2) v_{1}^{*}(x)+(1 / 2) v_{2}^{*}(x)\right]} \\
& \cdot d x \leq \frac{1}{2} J\left(h_{1}^{\circ}\right)+\frac{1}{2} J\left(h_{2}^{\circ}\right),
\end{aligned}
$$

with equality if and only if $h_{1}^{\circ}=h_{2}^{\circ}$. Therefore,

$$
\delta J(f, h) J\left(h^{\circ}\right)^{p / n} \leq \delta J\left(f, h_{1}\right) J\left(h_{1}^{\circ}\right)^{p / n}
$$

is the contradiction that would arise if it was the case that $h_{1} \neq h_{2}$.

The unique function whose existence is guaranteed by Proposition 9 will be denoted by $T_{p} f$, and will be called the $p$-Petty body of log-concave function $f$ (or the $\lambda$-Petty function). The polar function of $T_{p} f$ will be denoted by $T_{p}^{\circ} f$, rather than $\left(T_{p} f\right)^{\circ}$. For $f \in \mathscr{A}$ and $p>0$, the log-concave function $T_{p} f$ is defined by

$$
\begin{aligned}
& G_{p}^{(1)}(f)=\delta J\left(f, T_{p} f\right), \\
& J\left(T_{p}^{\circ} f\right)=c_{n} .
\end{aligned}
$$

Lemma 10. If $p>0$ and $f \in \mathscr{A}$, then for $A \in S L(n)$,

$$
T_{p}(f \circ A)=T_{p} f \circ A .
$$


Proof. From the definition of $T_{p}$ and Lemma 7 ,

$$
\delta J\left(f, T_{p} f\right)=G_{p}^{(1)}(f)=G_{p}^{(1)}(f \circ A)=\delta J\left(f \circ A, T_{p}(f \circ A)\right),
$$

Lemma 6 deduces

$$
\delta J\left(f, T_{p} f\right)=\delta J\left(f \circ A, T_{p}(f \circ A)\right)=\delta J\left(f, T_{p}(f \circ A) \circ A^{-1}\right) .
$$

The uniqueness of Proposition 9 ensures that $T_{p} f=$ $T_{p}(f \circ A) \circ A^{-1}$.

By the Blaschke-Santaló inequality, we obtain the following affine isoperimetric inequality for the functional geominimal surface area.

Theorem 11. Let $f \in \mathscr{A}^{\prime}$ and $p>0$. If $f$ has its barycenter at 0 , then

$$
J(f)^{p / n} G_{p}^{(1)}(f) \leq c_{n}^{p / n}\left(n J(f)+\int_{\mathbb{R}^{n}} f \log f d x\right)
$$

with equality if $T_{p} f(x)=f(x)$ and $f(x)=c e^{-\left(\|A x\|^{2} / 2\right)}$ for $A \epsilon$ $S L(n)$ and $c>0$.

Proof. Taking $g=f$ in (49), together with (33), we have,

$$
c_{n}^{p / n} G_{p}^{(1)}(f) \leq \delta J(f, f) J\left(f^{\circ}\right)^{p / n}=\left(n J(f)+\int_{\mathbb{R}^{n}} f \log f d x\right) J\left(f^{\circ}\right)^{p / n},
$$

i.e.,

$$
c_{n}\left(\frac{G_{p}^{(1)}(f)^{n}}{\left(n+(1 / J(f)) \int_{\mathbb{R}^{n}} f \log f d x\right)^{n} J(f)^{n-p}}\right)^{1 / p} \leq J(f) J\left(f^{\circ}\right) .
$$

By Blaschke-Santaló inequality (20) and the above inequality, we have

$$
\left(\frac{G_{p}^{(1)}(f)^{n}}{\left(n+(1 / J(f)) \int_{\mathbb{R}^{n}} f \log f d x\right)^{n} J(f)^{n-p}}\right)^{1 / p} \leq c_{n} .
$$

This is the desired inequality.

To obtain the equality condition, first assume that $T_{p} f=f$. Formula (77) tells us that

$$
G_{p}^{(1)}(f)=\delta J(f, f) \text { and } J\left(f^{\circ}\right)=c_{n} .
$$

This shows that there is equality in (81). From the condition of Blaschke-Santaló inequality, we known that there exists a positive definite matrix $A$ and $c>0$ such that, a.e. in $\mathbb{R}^{n}$,

$$
f(x)=c e^{-\frac{\|A x\|^{2}}{2}} .
$$

Therefore, we obtain the equality condition, namely, $T_{p} f=f$ and $f(x)=c e^{-\left(\|A x\|^{2} / 2\right)}$.

\section{Data Availability}

No data were used to support this study.

\section{Conflicts of Interest}

The authors declare no conflict of interest.

\section{Authors' Contributions}

All authors contributed equally to this work. All authors have read and agreed to the published version of this manuscript. The second author is the corresponding author.

\section{Acknowledgments}

N. Fang was supported in part by China Postdoctoral Science Foundation (No. 2019M651001). J. Yang was supported in part by NSFC (No. 11971005).

\section{References}

[1] R. Gardner, "The Brunn-Minkowski inequality," Bulletin of the American Mathematical Society, vol. 39, no. 3, pp. 355405, 2002.

[2] H. Brascamp and E. Lieb, "On extensions of the BrunnMinkowski and Prékopa-Leindler theorems, including inequalities for log concave functions, and with an application to the diffusion equation," Journal of Functional Analysis, vol. 22, no. 4, pp. 366-389, 1976.

[3] M. Fradelizi and M. Meyer, "Some functional inverse Santaló inequalities," Advances in Mathematics, vol. 218, no. 5, pp. 1430-1452, 2008.

[4] B. Klartag and V. D. Milman, "Geometry of log-concave functions and measures," Geometriae Dedicata, vol. 112, no. 1, pp. 169-182, 2005.

[5] L. Leindler, "On a certain converse of Hölder inequality II," Acta Scientiarum Mathematicarum, vol. 33, pp. 217-223, 1972.

[6] A. Prékopa, "Logarithmic concave measures with applications to stochastic programming," Acta Scientiarum Mathematicarum, vol. 32, pp. 335-343, 1971.

[7] A. Prékopa, "On logarithmic concave measures and functions," Acta Scientiarum Mathematicarum, vol. 34, pp. 335343, 1973.

[8] A. Prékopa, "New proof for the basic theorem of logconcave measures Hungarian," Alkalmazott Matematikai Lapja, vol. 1, pp. 385-389, 1975.

[9] D. Alonso-Gutiérrez, "A reverse Rogers-Shephard inequality for log-concave functions," The Journal of Geometric Analysis, vol. 29, no. 1, pp. 299-315, 2019.

[10] D. Alonso-Gutiérrez, S. Artstein-Avidan, B. G. Merino, C. H. Jiménez, and R. Villa, "Rogers-Shephard and local Loomis- 
Whitney type inequalities," Mathematische Annalen, vol. 374, no. 3-4, pp. 1719-1771, 2019.

[11] D. Alonso-Gutiérrez, J. Bernues, and B. G. Merino, “An extension of Berwald's inequality and its relation to Zhang's inequality," Journal of Mathematical Analysis and Applications, vol. 486, no. 1, p. 123875, 2020.

[12] D. Alonso-Gutiérrez, J. Bernués, and B. G. Merino, "Zhang's inequality for log-concave functions," https://arxiv.org/abs/ 1810.07507 .

[13] D. Alonso-Gutiérrez, B. G. Merino, C. H. Jiménez, and R. Villa, "Rogers-Shephard inequality for log-concave functions," Journal of Functional Analysis, vol. 271, no. 11, pp. 3269-3299, 2016.

[14] S. Artstein-Avidan, B. Klartag, and V. Milman, "The Santaló point of a function, and a functional form of the Santaló inequality," Mathematika, vol. 51, no. 1-2, pp. 33-48, 2004.

[15] S. Artstein-Avidan, B. Klartag, C. Schütt, and E. M. Werner, "Functional affine-isoperimetry and an inverse logarithmic Sobolev inequality," Journal of Functional Analysis, vol. 262, no. 9, pp. 4181-4204, 2012.

[16] S. Artstein-Avidan and V. Milman, "The concept of duality in convex analysis, and the characterization of the Legendre transform," Annals of Mathematics, vol. 169, no. 2, pp. 661674, 2009.

[17] S. Artstein-Avidan and B. A. Slomka, "A note on Santaló inequality for the polarity transform and its reverse," Proceedings of the American Mathematical Society, vol. 143, no. 4, pp. 1693-1704, 2015.

[18] S. Artstein-Avidan and B. A. Slomka, "Functional covering numbers," Journal of Geometric Analysis, 2019.

[19] K. Ball, "Logarithmically concave functions and sections of convex sets in $\$ \mathrm{R} \wedge\{\mathrm{n}\} \$$," Studia Mathematica, vol. 88 , no. 1, pp. 69-84, 1988.

[20] M. Barchiesia, G. M. Caprianib, N. Fusco, and G. Pisante, "Stability of Pólya-Szegő inequality for log-concave functions," Journal of Functional Analysis, vol. 267, no. 7, pp. 22642297, 2014.

[21] F. Barthe, K. J. Böröczky, and M. Fradelizi, "Stability of the functional forms of the Blaschke-Santaló inequality," Monatshefte für Mathematik, vol. 173, no. 2, pp. 135-159, 2014.

[22] U. Caglar, M. Fradelizi, O. Guedon, J. Lehec, C. Schütt, and E. M. Werner, "Functional versions of $L_{p}$-affine surface area and entropy inequalities," International Mathematics Research Notices, vol. 2016, no. 4, pp. 1223-1250, 2016.

[23] U. Caglar and E. M. Werner, "Divergence for $s$-concave and $\log$ concave functions," Advances in Mathematics, vol. 257, pp. 219-247, 2014.

[24] U. Caglar and E. M. Werner, "Mixed $f$-divergence and inequalities for log-concave functions," Proceedings of the London Mathematical Society, vol. 110, no. 2, pp. 271-290, 2015.

[25] U. Caglar and D. Ye, "Affine isoperimetric inequalities in the functional Orlicz-Brunn-Minkowski theory," Advances in Applied Mathematics, vol. 81, pp. 78-114, 2016.

[26] M. Fradelizi and M. Meyer, "Some functional forms of Blaschke-Santaló inequality," Mathematische Zeitschrift, vol. 256, no. 2, pp. 379-395, 2007.

[27] Q. Huang and A. Li, "The functional version of the Ball inequality," Proceedings of the American Mathematical Society, vol. 145, no. 8, pp. 3531-3541, 2017.
[28] J. Lehec, "A direct proof of the functional Santaló inequality," Comptes Rendus Mathematique, vol. 347, no. 1-2, pp. 55-58, 2009.

[29] J. Lehec, "Partitions and functional Santaló inequalities," Archiv der Mathematik, vol. 92, no. 1, pp. 89-94, 2009.

[30] B. Li, C. Schütt, and E. M. Werner, "Floating functions," Israel Journal of Mathematics, vol. 231, no. 1, pp. 181-210, 2019.

[31] Y. Lin, “Affine Orlicz Pólya-Szegö principle for log-concave functions," Journal of Functional Analysis, vol. 273, no. 10, pp. 3295-3326, 2017.

[32] V. Milman and L. Rotem, "Mixed integrals and related inequalities," Journal of Functional Analysis, vol. 264, no. 2, pp. 570-604, 2013.

[33] F. Mussnig, "Volume, polar volume and Euler characteristic for convex functions," Advances in Mathematics, vol. 344, pp. 340-373, 2019.

[34] L. Rotem, "Support functions and mean width for $\alpha$-concave functions," Advances in Mathematics, vol. 243, pp. 168-186, 2013.

[35] A. Colesanti and I. Fragalà, "The first variation of the total mass of log-concave functions and related inequalities," Advances in Mathematics, vol. 244, pp. 708-749, 2013.

[36] C. M. Petty, "Geominimal surface area," Geometriae Dedicata, vol. 3, pp. 77-97, 1974.

[37] E. Lutwak, "The Brunn-Minkowski-Firey theory II," Advances in Mathematics, vol. 118, no. 2, pp. 244-294, 1996.

[38] D. Ye, "Lp geominimal surface areas and their inequalities," International Mathematics Research Notices, vol. 9, pp. 24652498, 2015.

[39] D. Ye, "Dual Orlicz-Brunn-Minkowski theory: dual Orlicz $L_{\varphi}$ affine and geominimal surface areas," Journal of Mathematical Analysis and Applications, vol. 443, no. 1, pp. 352-371, 2016.

[40] D. Ye, B. Zhu, and J. Zhou, “The mixed \$Lp\$ geominimal surface areas for multiple convex bodies," Indiana University Mathematics Journal, vol. 64, no. 5, pp. 1513-1552, 2015.

[41] B. Zhu, N. Li, and J. Zhou, "Isoperimetric inequalities for $L_{p}$ geominimal surface area," Glasgow Mathematical Journal, vol. 53, no. 3, pp. 717-726, 2011.

[42] B. Zhu, J. Zhou, and W. Xu, "Lp mixed geominimal surface area," Journal of Mathematical Analysis and Applications, vol. 422, no. 2, pp. 1247-1263, 2015.

[43] T. Rockafellar, Convex Analysis, Princeton University Press, Princeton, 1970.

[44] L. Rotem, "On the mean width of log-concave functions," in Geometric Aspects of Functional Analysis, B. Klartag, S. Mendelson, and V. Milman, Eds., vol. 2050 of Lecture Notes in Mathematics, Springer, Berlin, Heidelberg, 2012.

[45] E. Lutwak, "The Brunn-Minkowski-Firey theory I Mixed volumes and the Minkowski problem," Journal of Differential Geometry, vol. 38, pp. 131-150, 1993.

[46] D. Cordero-Erausquin and B. Klartag, "Moment measures," Journal of Functional Analysis, vol. 268, no. 12, pp. 3834$3866,2015$. 